\title{
Reduction of low-frequency noise in magnetic induction tomography systems
}

\author{
H. Scharfetter, S. Issa \\ Institute of Medical Engineering, Graz University of Technology, Kronesgasse 5, 8010 Graz, Austria
}

\begin{abstract}
Magnetic induction tomography (MIT) aims at the contact-less imaging of the electrical conductivity and related pathophysiological processes. Systems operating at frequencies below $1 \mathrm{MHz}$ are desirable but suffer from poor SNR which can be dramatically impaired by mechanical vibrations. We present an algorithm which suppresses vibration noise in the imaginary part of the data and improves the SNR up to $35 \mathrm{~dB}$.
\end{abstract}

Keywords- Magnetic induction tomography, conductivity imaging, noise suppression, signal to noise ratio.

\section{INTRODUCTION}

Magnetic induction tomography (MIT) [1],[2],[3] is a contact-less imaging modality which maps the electrical conductivity within a 3-D body. MIT requires an alternating magnetic excitation field to be coupled from an array of transmit coils (TXC) to the object. Changes of the conductivity cause a field perturbation which is sensed in form of induced voltages in an array of receiver coils (RXC). Images are usually reconstructed from voltage changes $\Delta \mathbf{v}$ between different states of the object (e. g. during movements or when changing the excitation frequency), whereas the components of $\Delta \mathbf{v}$ stem from all possible independent TX-RX combinations. The image reconstruction problem has been described elsewhere and is out of the scope of this paper. Suffices to say that the reconstruction involves a highly ill-posed problem which is very sensitive to noise in the data, therefore high SNR is mandatory.

Most MIT hardware so far developed for medical investigation uses frequencies of several $\mathrm{MHz}$ to provide high sensitivity in low conductivity tissues, i.e. $20 \mathrm{MHz}$, [2], $10 \mathrm{MHz}[1,4]$. However, measurements in the $\beta$ dispersion range below $1 \mathrm{MHz}$ are desirable because many pathological processes are reflected there [5]. Due to the poor SNR, however, this represents a real challenge. The lowest frequency so far tried was found in [6] $(11.6 \mathrm{kHz})$. We have developed a fast parallel multi-frequency imaging system which operates from $50 \mathrm{kHz}$ to $1 \mathrm{MHz}$ [7]. It comprises 16 RXC and 16 TXC and allows frequency differential imaging [8], whereas $8+8$ coils can be operated in single shot mode. However, due to the the low sensitivity vibrations and thermal drifts cause significant errors [7]. This paper describes a method for eliminating such errors.

\section{METHODS}

\section{A. System description}

The Graz MIT Mk2 system consists of two ring shaped coil arrays with 8 solenoid TXC and 8 RXC on each ring.

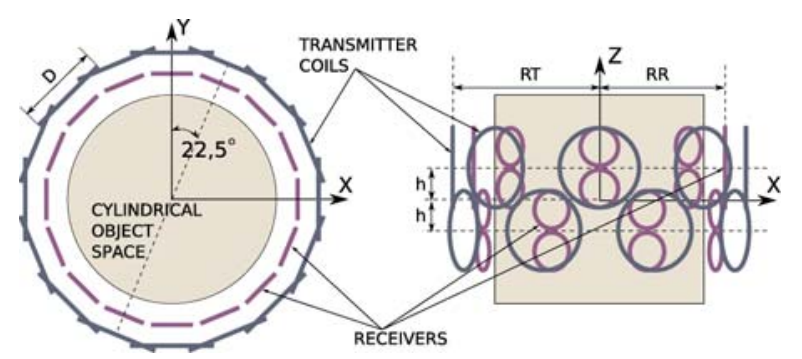

Fig. 1: Geometry of the coil array. The parameters RT, RR and h are variable in our mounting structure. $\mathrm{D}=100 \mathrm{~mm}$.

The coils are arranged in a zig-zag pattern around the perimeter (fig. 1). Each RXC is a planar gradiometes placed in front of a TXC, facing the center of the ring and adjusted so as to cancel the net magnetic flux produced by the TXC.

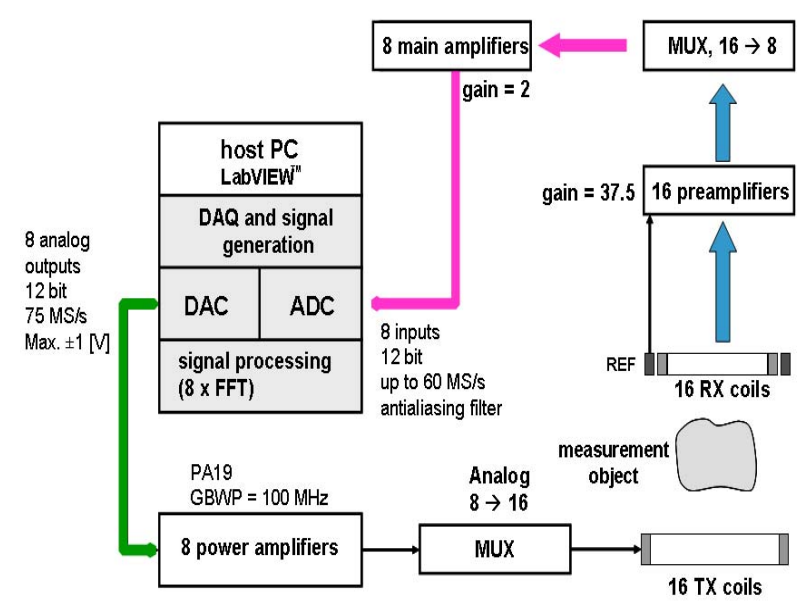

Fig. 2: Schematic block diagram of the Graz Mk2 MIT system

In fig. 2 the block diagram of the complete hardware is shown. The excitation signals are generated as multisinus patterns by an 8-channel DAC board and fed to 8 power 
amplifiers (PAs). To drive all 16 TXCs a $8 \rightarrow 16$ multiplexer is provided. The response voltages from the 16 RXC are preamplified and demultiplexed $16 \rightarrow 8$ before being digitized by an 8-channel ADC board. As the 8 TXCs are fed simultaneously they must be encoded by splitting the carrier frequencies into 8 closely neighboring sub-frequencies. The amplitude and phase of each TXC/RXC signal is then extracted by the FFT.

\section{B. Systematic errors}

To extract the complex conductivity information with the correct phase, the true EMFs in the RXCs should be demodulated with respect to a voltage induced in a reference coil by the primary field only. However, in the real system the digital demodulation is done with respect to the phase of the local oscillator (LO) of the signal processing unit. As the signal travels through many stages to the TXC and through the receiver chain it suffers systematic phase changes so that the true phase relationship is lost. We analyze the problem according to fig. 3. The basic coordinate system is the physical frame $\left(\operatorname{Re}_{\mathrm{TX}}, \mathrm{Im}_{\mathrm{TX}}\right)$ in which the EMF induced in an RXC by the primary magnetic flux of a TXC is purely real. Only changes of voltages are depicted.

Assumption 1: In weak conductors (biological tissues) and below several $\mathrm{MHz}$ the real part $\sigma$ of the conductivity causes only an imaginary change $s$ in the frame $\left(\operatorname{Re}_{\mathrm{TX}}\right.$, $\mathrm{Im}_{\mathrm{TX}}$ ). Movements of the TXC with respect to the RXC cause a modulation of the mutual inductance and hence of the induced voltage. The resulting modulation signal $\mathrm{m}$ is purely real in $\left(\operatorname{Re}_{\mathrm{TX}}, \mathrm{Im}_{\mathrm{TX}}\right)$. Given the need for resolving less than 1 ppm of the primary voltage mechanical vibrations thus can heavily deteriorate the SNR of the real part while the imaginary part remains unaffected [10]. Aiming only at the reconstruction of $\sigma$ the further analysis can be limited to the imaginary signal s.

Assumption 2: The signal $\mathrm{s}$ is assumed to be much weaker modulated by the movement than $m$ so that $s$ can be considered as unmodulated in the further analysis.

In practice, the excitation waveform is generated by the $\mathrm{LO}$ which defines the $\mathrm{LO}$ frame $\left(\mathrm{Re}_{\mathrm{LO}}, \mathrm{Im}_{\mathrm{LO}}\right)$ for demodulation. The waveform is sent through the TX chain (ADC, PA, TXC) and suffers a systematic phase shift $\theta_{1}$ before showing up in the physical frame $\left(\operatorname{Re}_{\mathrm{TX}}, \mathrm{Im}_{\mathrm{TX}}\right)$. Then both $\mathrm{s}$ and $\mathrm{m}$ travel further through the parasitic LRC network of the RX coils and suffer a phase shift $\varphi$ and finally the signals encounter a further shift $\theta_{2}$ caused by the following amplifiers and the DAC. When demodulating these rotated replicas s' and m' with respect to the LO frame
$\left(\mathrm{Re}_{\mathrm{LO}}, \mathrm{Im}_{\mathrm{LO}}\right)$ a strong spurious imaginary modulation $\mathrm{m}_{\mathrm{s}}$ appears although in reality there is none in the physical frame (assumption 2). The imaginary signal $s_{s}$ is the projection of $s$ ' to the axis $\operatorname{Im}_{\mathrm{LO}}$ and does not reflect correctly the magnitude of the true signal s. Hence mechanical vibrations can heavily deteriorate the SNR of the measured imaginary signal [7]. Also other errors which affect mainly the true real part (moving metallic parts, thermal drifts of the RXC [5]) project into $\operatorname{Im}_{\mathrm{LO}}$.

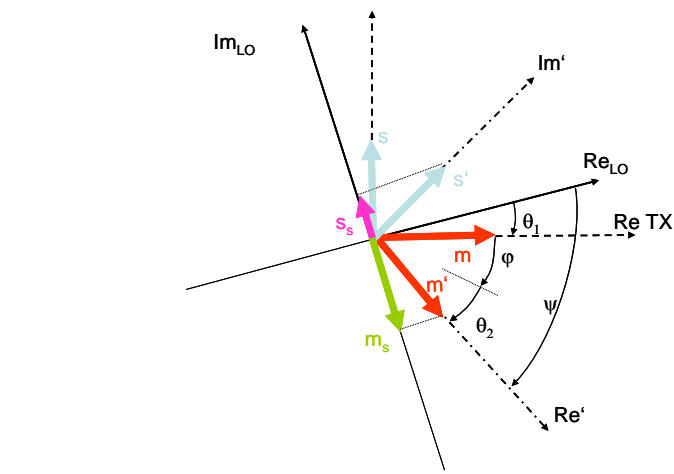

Fig. 3: phase rotations along the signal path

\section{Phase correction}

In order to avoid the above spurious projections we must back-rotate the signals by $\psi=\left(\theta_{1}+\theta_{2}+\varphi\right)$ whereas $\theta_{1}$ in previous concepts was provided by a separate reference coil which picked up the primary magentic flows from all TXC at once [7]. In the new approach the correction is achieved by rotating the signals until the spurious modulation $m_{S}$ vanishes, i. e. $\operatorname{Var}\left(\mathrm{S}_{\mathrm{S}}\right)$ is minimum. This rotation accounts for all error phases $\varphi, \theta_{2}, \theta_{1}$ without the need for an extra reference channel. Setting: $\mathrm{z}:=\operatorname{Im}_{\mathrm{LO}}, \mathrm{x}:=\mathrm{m}$; $\mathrm{y}=\mathrm{s}$ ' the imaginary part in the LO frame is

$$
z=-x \sin \Psi+y \cos \Psi
$$

The variance of this imaginary part is

$$
\begin{aligned}
& \operatorname{Var}(z)=E\left\{(z)^{2}\right\}=E\left\{(y \cos \Psi-x \sin \Psi)^{2}\right\}= \\
& =E\left\{y^{2} \cos ^{2} \Psi\right\}+E\left\{x^{2} \sin ^{2} \Psi\right\}-2 E(x y \cos \Psi \sin \Psi)= \\
& =\cos ^{2} \Psi \operatorname{Var}(y)+\sin ^{2} \Psi \operatorname{Var}(x)-2 \cos \Psi \sin \Psi \operatorname{Cov}(x, y)
\end{aligned}
$$

With $\sin \Psi=a ; \quad \cos \Psi=\sqrt{1-a^{2}}$ and setting $\mathrm{f}:=\operatorname{Var}(\mathrm{z})$, $U:=\operatorname{Var}(x), V:=\operatorname{Var}(y), W:=\operatorname{Cov}(x, y)$ eq. (2) becomes: 


$$
f(a)=\left(1-a^{2}\right) V+a^{2} U-2 a \sqrt{1-a^{2}} W
$$

Minimization of $\operatorname{Var}(\mathrm{z})$ with respect to $\psi$ yields:

$$
\min _{\Psi} f \rightarrow \frac{d}{d \psi} f=0=\frac{d f}{d a} \frac{d a}{d \psi}=\frac{d}{d a} f \cos (\psi)
$$

Excluding the trivial case $|\psi|=\pi / 2$ (4) is equivalent to:

$$
\begin{aligned}
& U-V-W\left(\frac{\sqrt{1-a^{2}}}{a}-\frac{a}{\sqrt{1-a^{2}}}\right)=0 \\
& \rightarrow{ }_{2} \cot (\psi)_{1}=-\left(\frac{U-V}{2 W}\right) \pm \sqrt{\left(\frac{U-V}{2 W}\right)^{2}+1}
\end{aligned}
$$

(6)

from which we pick that $\psi$ which minimizes $\operatorname{Var}(\mathrm{z})$.

\section{Experimental evaluation}

$U, V, W$ were calculated from 15 calibration frames during which we introduced vibrations (sinusoidal, 20 $\mathrm{Hz}$ ) in the coil system with a loudspeaker so as to get sufficient variance in the data. During acquisition of the following 26 measurement frames the vibration was stopped. The correction angles were calculated only from the calibration data and then applied to the measurement data. DAQ parameters: 12 bit, 7.5 MSamples/s, 20 ms window length. Injected current: Two superimposed sinusoids, $0.85 \mathrm{~A}_{\mathrm{pp}} @ 200 \mathrm{kHz}$ and $0.31 \mathrm{~A}_{\mathrm{pp}} @ 450 \mathrm{kHz}$, subfrequency spacing $300 \mathrm{~Hz}$.

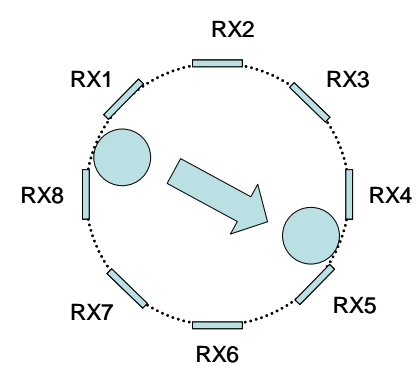

Fig. 4: Experiment for the determination of the SNR. The blue circles illustrate the saline bottle which was shifted after 13 frames to the opposite side of the upper RX ring. Top view.

The equivalent spectral noise voltage density (ENVD) was first determined with the empty coil system and the PA switched off. Then we loaded the system with a plastic cylinder filled with saline (8 $\mathrm{S} / \mathrm{m}$, Ø $7 \mathrm{~cm}$, height $=10 \mathrm{~cm}$, orientation in $\mathrm{z}$-axis) placed as shown in fig. 4. After 13 frames the object was shifted as indicated by the arrow.

\section{RESULTS}

With the PA switched off the ENVD was always less then $2 \mathrm{nV} / \mathrm{sqrt}(\mathrm{Hz})$ which is close to the value expected from the pre-amplifiers $(1.5 \mathrm{nV} / \mathrm{sqrt}(\mathrm{Hz}))$.

Fig. 5 shows the ENVD of all $8 \mathrm{RXC}$ and receiving from TXC6 after switching on the PA. The noise is highest for RXC6 and the neighboring coils RXC5 and RXC7 which have high magnetic coupling with TXC6 and thus are very sensitive to vibrations. After phase correction the ENVD decreased by a factor of 21 in RXC6.

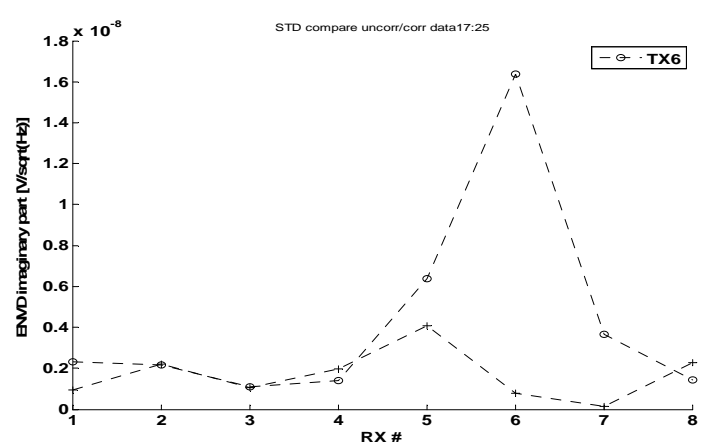

Fig. 5: Comparison of the equivalent noise voltage density between uncorrected (o) and corrected (+) data of TX channel 6

Most channels improved significantly, some by up to a factor of 100 while only in 4 channels the noise increased slightly. Fig 6 quantifies the SNR (the ratio between the std of the signal change due to the object shift and the standard deviation of the noise voltage) before (A) and after (B) correction (please note the change of the colorbar scaling). In several channels (e. g. TXC5/RXC5) the improvement is more than $30 \mathrm{~dB}$ as shown in fig. 7 for both frequencies. Channels with still low SNR (e. g. TXC2/RXC3) are only those which inherently exhibit poor sensitivity to the chosen locations of the test object. Typically the SNR is high at the crossings of rows/columns $1,4,5$ and 8 , 
respectively, because these reflect those coil combinations which were most close to the object.
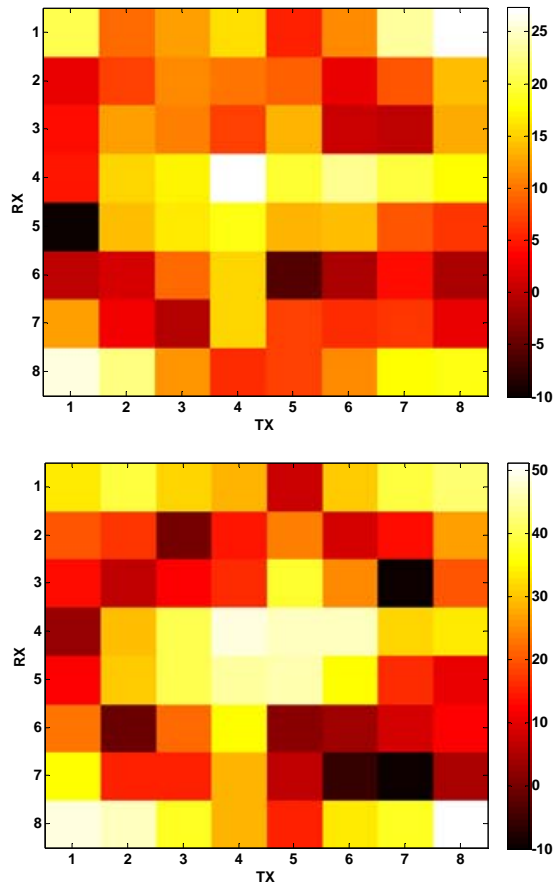

A

\section{B}

Fig. 6: SNR [dB] of the uncorrected (A) and corrected (B) system. Please note the different scales on the colorbar.
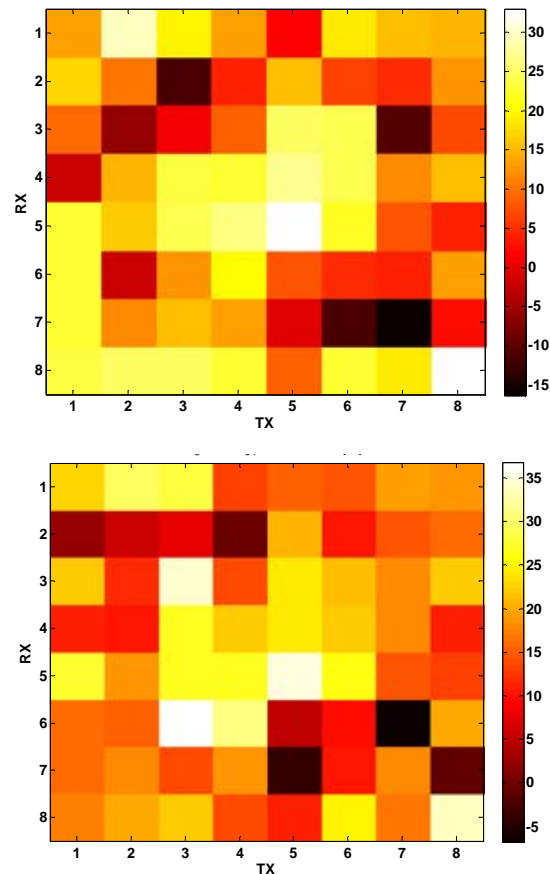

Fig. 7: gain of SNR [dB] due to correction at 200kHz (A) and $450 \mathrm{kHz}$ (B). Please note the different scales on the colorbar.

\section{Discussion}

The proposed algorithm provides an effective correction of the phase errors in the presented MIT system without extra reference channels. Vibrational low frequency noise could be reduced and SNR increased by up to $35 \mathrm{~dB}$. The gain of SNR between $\mathrm{f}_{1}=200$ and $\mathrm{f}_{2}=450 \mathrm{kHz}$ is about $5 \mathrm{~dB}$ due to the lower excitation current I at $\mathrm{f}_{2}$. Actually the expected gain of sensitivity is $I\left(f_{2}\right) / I\left(f_{1}\right) *\left(f_{2} / f_{1}\right)^{2}$ which yields $5.3 \mathrm{~dB}$. In an ideally symmetric system the SNR matrix (fig. 6) should be nearly symmetric but due to different vibration dynamics of different coils the symmetry is not perfect. The achieved system performance is sufficient for low contrast conductivity imaging such as in [8].

\section{AcKNowledgment}

This work was supported by the SFB project F32N18 granted by the Austrian Science Fund.

\section{REFERENCES}

1. Griffiths H, et al (1999) Magnetic induction tomography. A measuring system for biological tissues. Ann NY Acad Sci 873: 335-345

2. Korjenevsky A, et al (2000) Magnetic induction tomography: Experimental realization. Physiol Meas 21: 89-94

3. Peyton A J et al (1996) An overview of electromagnetic inductance tomography: Description of three different systems. Meas Sci Technol 7: 261-271

4. Vauhkonen M, et al (2008) A measurement system and image reconstruction in magnetic induction tomography Physiol Meas 29: S445-S454

5. Scharfetter H, Casañas R and Rosell J (2003) Biological Tissue Characterization by Magnetic Induction Spectroscopy (MIS): Requirements and Limitations IEEE Trans Biomed Eng 50: 870-80

6. Karbeyaz UB, Gencer NG (2003) Electrical conductivity imaging via contactless measurements: an experimental study. IEEE Trans Med Imaging 22: 627 - 635.

7. $\mathrm{H}$ Scharfetter et al (2008) Hardware for quasi-single-shot multifrequency magnetic induction tomography (MIT): the Graz Mk2 system. Physiol. Meas 29: S431-S443

8. Brunner P, et al. (2006) Reconstruction of the shape of conductivity spectra using differential multi-frequency magnetic induction tomography. Physiol Meas 27: S237-S248 
Address of the corresponding author:

- $\quad$ Author:

- Institute:

- Street:

- City:

- Country:

- Email:

Hermann Scharfetter

Inst. Of Medical Engineering

Kronesgasse 5

hermann.scharfetter@tugraz.at 